\title{
Pseudoaneurysm Formation at the Rupture Site of a Middle Cerebral Artery Aneurysm
}

\author{
-Case Report- \\ Mitsunobu IDE, Tomonori KOBAYASHI, Yoshinori TAMAnO, Shinji HAgIWARA, \\ Noriko TANAKA, and Hirotsune KAWAMURA
}

Department of Neurosurgery, Tokyo Women's Medical University Daini Hospital, Tokyo

\begin{abstract}
A 42-year-old man suffered subarachnoid hemorrhage manifesting as sudden severe headache one month before admission. On admission, his headache had subsided and he had no neurological deficits. Cerebral angiography demonstrated an aneurysm originating from the bifurcation of the right middle cerebral artery. The aneurysm was irregular, with a snowman-like shape. Neck clipping of the aneurysm was carried out through a right pterional approach. Intraoperatively, a red, pulsating sac mimicking a blood clot (the snowman's "head") was located over the yellowish, thick-walled portion of the aneurysm. Exploration around the aneurysm detached the red sac from the thick-walled portion of the aneurysm. There was a small tear in the apex of the thick-walled aneurysm sac. A Sugita clip was applied to the neck of the true aneurysm. The postoperative course was uneventful and he was discharged one month later without neurological deficits. Histological examination of the red-colored sac showed the features of pseudoaneurysm. The red sac may have been a pseudoaneurysm covering the rupture site of the true aneurysm.
\end{abstract}

Key words: pseudoaneurysm, false aneurysm, cerebral aneurysm, subarachnoid hemorrhage, clipping

\section{Introduction}

Blebs commonly occur at the rupture site of true cerebral aneurysms. Reorganization of the blood clot covering the rupture site results in extension of the lumen, which may appear as a bleb on angiography. ${ }^{1)}$ Such a bleb might be considered as a small pseudoaneurysm. Autopsy studies have revealed the formation of large pseudoaneurysms over the rupture sites of true aneurysm sac, ${ }^{3,5)}$ but there are few clinical reports of such pseudoaneurysms. ${ }^{2)}$ We treated a patient who presented with subarachnoid hemorrhage associated with a large pseudoaneurysm covering a true aneurysm of the middle cerebral artery.

\section{Case Report}

A 42-year-old male suddenly experienced severe headache soon after scuba diving near a tropical island on August 27, 2000. He visited a local hospital

Received January 20, 2003; Accepted April 3, 2003 on September 8. Computed tomography (CT) demonstrated subarachnoid hemorrhage. He was air-lifted back to Tokyo and admitted to our facility on September 26. On admission, his headache had subsided and he had no neurological deficits. He had no past history of head trauma. Cerebral angiography demonstrated an aneurysm originating from the bifurcation of the right middle cerebral artery (Fig. 1). The aneurysm was irregular in shape, with a snowman-like appearance. The distal portion of the aneurysm (snowman's head) showed delayed opacification and delayed clearance of the contrast material. The distal middle cerebral artery showed segmental angiospasm. Three-dimensional CT angiography demonstrated a constriction of the aneurysm sac (Fig. 2).

The patient underwent surgery on October 2, 2000. Neck clipping of the aneurysm was carried out through the right pterional approach. Dissection of the sylvian fissure initially exposed a red, pulsating sac mimicking a blood clot. The sac was tightly adherent to the frontal and temporal lobes and half of the sac was buried in the base of the frontal lobe 

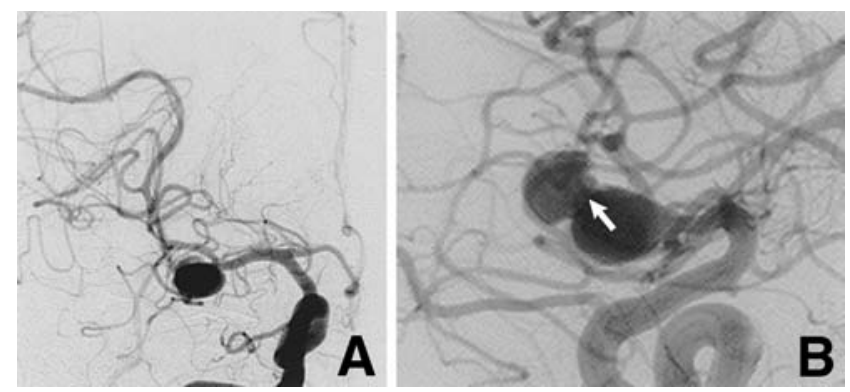

Fig. 1 Preoperative right internal carotid angiograms, anteroposterior (A) and lateral views (B), demonstrating a right middle cerebral artery aneurysm. The apex of the true aneurysm sac (arrow) projects into the faintly opacified pseudoaneurysm cavity.

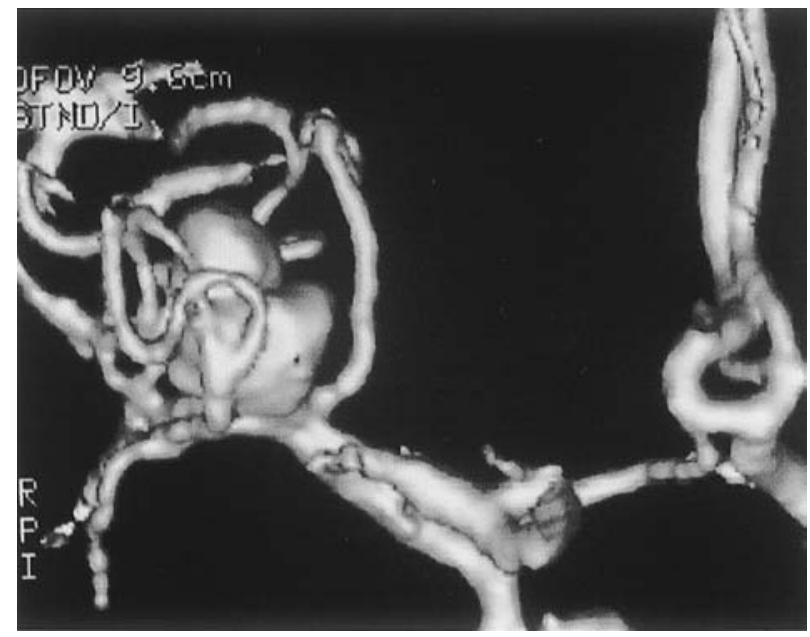

Fig. 2 Three-dimensional computed tomography angiogram demonstrating the snowman-like appearance of the aneurysm.

(Fig. 3A). The adjacent cerebral surface contained yellowish pigmentation, indicating previous subarachnoid hemorrhage. Opening of the sylvian fissure had caused premature bleeding from the red sac, so a temporary clip was applied to the horizontal $\left(M_{1}\right)$ segment of the right middle cerebral artery to control bleeding, which induced remarkable shrinkage of the red sac. Subsequent exploration around the aneurysm detached the sac from the thick-walled portion of the aneurysm (Fig. 3B). There was a small tear in the apex of the thickwalled portion of the aneurysm and a fragment of the red sac adhered to this portion of the aneurysm (Fig. 3C). These intraoperative observations suggested that the detached portion of the aneurysm might be a pseudoaneurysm, covering the rupture site of the true aneurysm. After confirmation that the neck
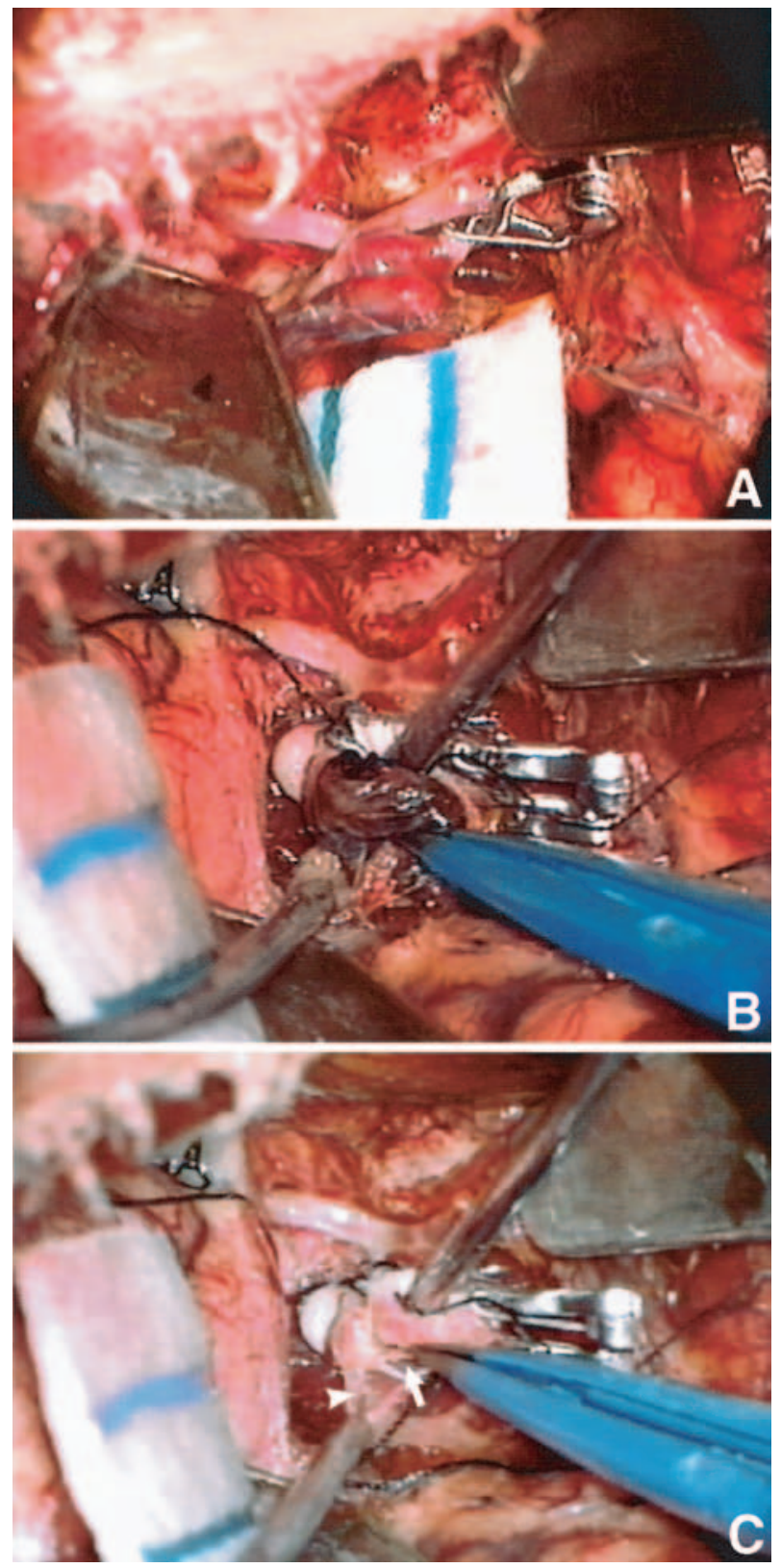

Fig. 3 Intraoperative photographs. (A) A red, pseudoaneurysm sac is tightly adherent to the frontal and temporal lobes and half of the sac is buried in the base of the frontal lobe. (B) The red sac is detached from the yellowish, thick-walled true aneurysm. (C) Note the small tear in the apex of the true aneurysm sac (arrow) and the fragment of the pseudoaneurysm sac (arrowhead) adhering to the rupture site of the true aneurysm.

of the true aneurysm arose from the middle cerebral artery bifurcation, a No. 10 Sugita clip was applied to the neck of the true aneurysm. The true aneurysm sac was partially resected. 

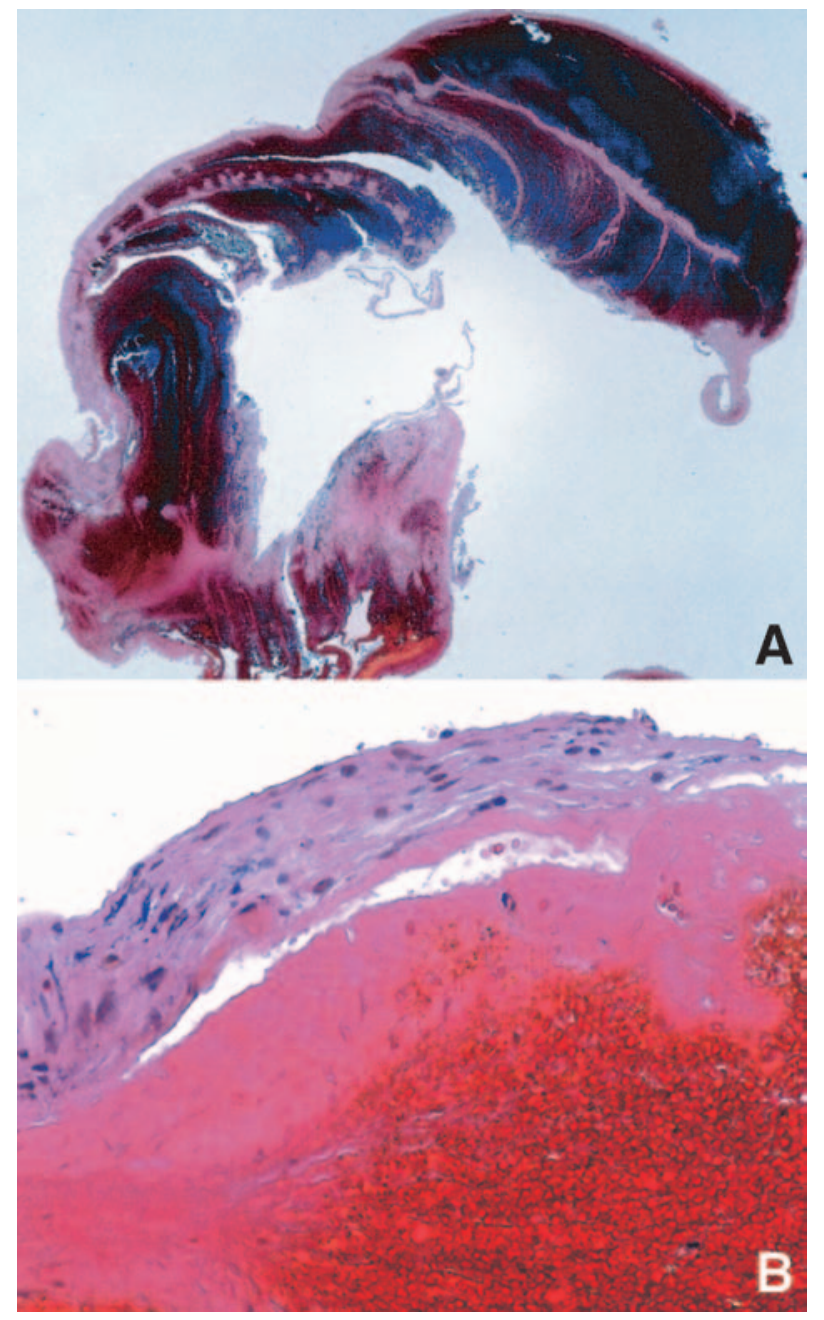

Fig. 4 Photomicrographs of the detached pseudoaneurysm. (A) The pseudoaneurysm wall consists of a fibrin layer with lymphoid infiltration. The fibrin layer is laminated and partially intermingled with thrombi. Fresh or old thrombi adhere to the inner surface of the aneurysm wall. Mixed fibrinous tissue and red blood cells form whirls in the pseudoaneurysm cavity. HE stain, $\times 20$. (B) Arachnoid tissue is attached to the outer surface of the pseudoaneurysm wall. Hemosiderin deposits can be seen in the pseudoaneurysm wall. HE stain, $\times 200$.

Histological examination showed the red sac was quite different from the true aneurysm. The sac wall consisted of a fibrin layer with a cellular component (Fig. 4). The fibrin layer was laminated and partially intermingled with blood clot elements. Hemosiderin deposition was noted in the wall. These histological features indicated that this portion of the excised lesion was a pseudoaneurysm. Fresh or old thrombi

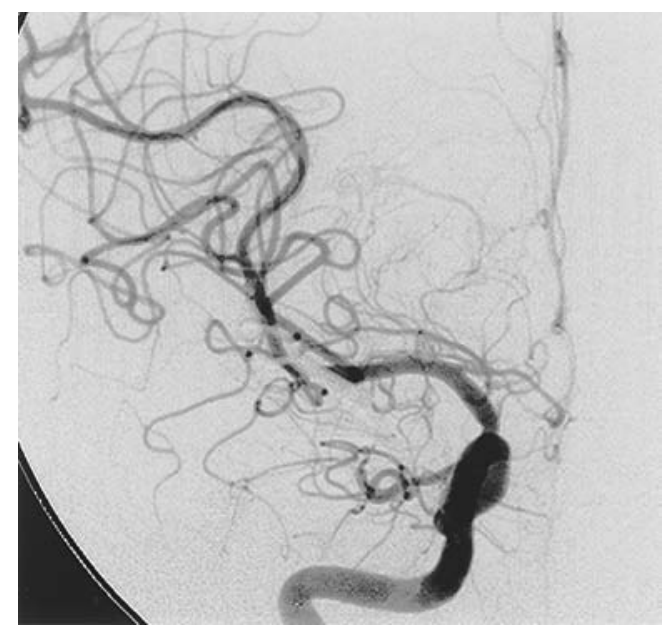

Fig. 5 Postoperative right internal carotid angiogram, anteroposterior view, confirming complete obliteration of the aneurysm.

were adherent to the inner surface of the pseudoaneurysm wall. Arachnoid tissue was attached to outer surface of the sac in some areas. In contrast, the wall of the true aneurysm was very thick and collagenous with atheromatous plaque.

Postoperatively, the patient's recovery was uneventful. Postoperative angiography showed obliteration of the aneurysm (Fig. 5). He was discharged one month later without neurological deficits and has since resumed his previous work.

\section{Discussion}

A pseudoaneurysm results from partial or complete rupture of the arterial wall, where the pulsatile hematoma formed from the extravasated blood is walled off by perivascular tissues and organization of a fibrinous coagula, with retention of functional continuity between the newly formed false lumen and the parent artery lumen.5) Traumatic false aneurysms are also occasionally reported, and form after rupture of the arterial wall due to mechanical stress.4) Our case is unique in that the pseudoaneurysm formed at the rupture site of the true aneurysm.

Autopsy studies have revealed large pseudoaneurysms formed over the rupture sites of true aneurysms, ${ }^{3,5)}$ but intraoperative observation of such pseudoaneurysms is unusual. ${ }^{2)}$ Angiographically irregularly-shaped aneurysms are frequently accompanied by fragile pseudoaneurysm-like cavities located at the rupture point, and a blood clot was observed adhering to the rupture point of the aneurysm during surgery. ${ }^{2)}$ Angiography has shown 
that some aneurysms rapidly enlarge after rupture. ${ }^{3)}$ Histological examination of these ruptured aneurysms with adjacent brain tissues at autopsy found a newly developed fibrinous layer, or pseudoaneurysm formation, covering the rupture site of the true aneurysm sac, and a massive blood clot around the ruptured true aneurysm containing an extension of the aneurysm lumen. Such extension of the lumen into the clot might appear as rapid enlargement of the aneurysm on angiography.

In the present case, the identification of the detached, red thin-walled portion of the aneurysm as a true pseudoaneurysm could be challenged. However, the angiographic configuration and the opacification pattern typical of a pseudoaneurysm were present. ${ }^{2)}$ Intraoperatively, the lesion was very fragile and even meticulous manipulation around the aneurysm induced bleeding. Histological examination showed the thin-walled sac had the characteristics of a pseudoaneurysm. ${ }^{3-5}$ We speculate that the pseudoaneurysm in our patient may have developed from the gradually reorganizing clot surrounding the site of rupture of the true aneurysm in the sylvian fissure. During the course, the blood clot covering the rupture site of the true aneurysm may have been dissolved from the inside by fibrinolysis, resulting in gradual enlargement of the pseudoaneurysm cavity. Reinforcement by the surrounding brain tissue including the arachnoid membrane may have promoted formation of a pseudoaneurysm.

In our patient, a month elapsed between the onset of subarachnoid hemorrhage and clipping of the aneurysm. The air-lift to Tokyo from the tropical island where he suffered the subarachnoid hemorrhage carried considerable risk. Fortunately, the patient did not experience rebleeding and his clinical course was satisfactory. His normal blood pressure may have favored this good outcome.

\section{References}

1) Byrne JV, Guglielmi G: Endovascular Treatment of Intracranial Aneurysms. Berlin, Springer, 1998, pp 7-9

2) Nomura M, Kida S, Uchiyama N, Yamashima T, Yoshikawa J, Yamashita J, Matsui O: Ruptured irregularly shaped aneurysms: pseudoaneurysm formation in a thrombus located at the rupture site. J Neurosurg 93: 998-1002, 2000

3) Ohara H, Suzuki J: [Clinicopathological study of cerebral aneurysms: origin, rupture, repair and growth]. No To Shinkei 29: 83-94, 1977 (Jpn, with Eng abstract)

4) Ohta M, Matsuno H: Proximal M2 false aneurysm after head trauma: case report. Neurol Med Chir (Tokyo) 41: 131-134, 2001

5) Stehbens WE: The pathology of intracranial arterial aneurysms and their complications, in Fox JL (ed): Intracranial Aneurysms. New York, Springer-Verlag, 1983, pp 272-357

Address reprint requests to: M. Ide, M.D., Department of Neurosurgery, Tokyo Women's Medical University Daini Hospital, 2-1-10 Nishiogu, Arakawa-ku, Tokyo 116-8567, Japan.

e-mail: idene@dnh.twmu.ac.jp 